\title{
Bandpass Negative Group Delay Demonstration on a VHF RLC Parallel Passive Lumped Circuit
}

\author{
Eric Jean Roy Sambatra1, Yves Constant Mombo Boussougou², Lucius Ramifidisoa ${ }^{3}$, \\ Jean-Paterne Kouadio4, Samuel Ngoho4, Antonio Jaomiary ${ }^{3}$, and Nour Mohammad Murad5
}

${ }^{1}$ Institut Supérieur de Technologie (ISTD), BP 509, Antsiranana 201, Madagascar

${ }^{2}$ Université des Sciences et Techniques de Masuku (USTM), BP 901, Franceville, Gabon

${ }^{3}$ Ecole Normale Supérieure pour l'Enseignement Technique (ENSET), BP O, Antsiranana 201, Madagascar

${ }^{4}$ Association Française de Science des Systèmes (AFSCET), Paris 75013, France

${ }^{5}$ Energy Lab, Network and Telecom team, Institut Universitaire de Technologie, University of La Reunion, Saint Denis 97715, France

Corresponding author: Eric Jean Roy Sambatra (e-mail: ericsambatra@gmail.com).

\begin{abstract}
This paper demonstrates the bandpass (BP) negative group delay (NGD) behavior of a passive RLC-parallel lumped network at very high frequencies (VHFs). After topological description, the BP NGD analysis is introduced. The BP NGD circuit is theoretically modelled by means of voltage transfer function (VTF) expression. The analytical equations illustrating the BP NGD specifications as the NGD center frequency, NGD value, NGD bandwidth and VTF attenuation are established in function of the R, L and $\mathrm{C}$ component parameters. To verify the relevance of the BP NGD analysis, a lumped circuit proof of concept (POC) is synthesized and designed. As prototype, the corresponding BP NGD circuit prototype of printed circuit board (PCB) is implemented in hybrid technology with SMD components. Then, quite innovated testing method allowing to extract the VTF is developed. As expected, the calculated, simulated and measured results are very well-correlated and showing BP NGD behavior of the POC. The calculated and simulated results with NGD value of $-8 \mathrm{~ns}$ (resp. $-12 \mathrm{~ns}$ ), attenuation of $-10 \mathrm{~dB}$ (resp. $-8 \mathrm{~dB}$ ) and bandwidth around $225 \mathrm{MHz}$ (resp. $240 \mathrm{MHz}$ ) NGD center frequency are obtained. In addition to the experimental validation, uncertainty analysis (UA) with respect to the BP NGD specifications is also studied in order to show the influence of quality and tolerance of lumped components. The theoretical formulas of NGD specification UAs in function of R, $\mathrm{L}$ and $\mathrm{C}$ tolerances are derived. The UAs with respect to $1 \%, 2 \%$ and $5 \%$ relative tolerances of $\mathrm{R}, \mathrm{L}$ and $\mathrm{C}$ components constituting the BP NGD circuit POC are discussed.
\end{abstract}

INDEX TERMS Negative group delay (NGD), Bandpass (BP) NGD circuit, NGD analysis, RLC parallel (RLCP) topology, lumped passive circuit, very high frequency (VHF), NGD specifications, uncertainty analysis (UA).

\section{INTRODUCTION}

$\mathbf{T}$ HE negative group delay (NGD) phenomenon was the first time observed with optical experimentation in 1980s [1-2]. The counterintuitive NGD experimental demonstration attracts the attention of curious physicists in twentieth century. This scientific curiosity leads to deeper investigation for the better understanding about the NGD effect meaning. Therefore, further theoretical investigations were conducted in the optical areas [3-6]. It was found that the NGD effect is materially linked to the negative group velocity (NGV) [3-4]. It is worth to note that the NGV effect are basically evaluated with wave propagating through a resonant and dispersive medium represented by a 1-D or 2-D or 3-D bulk materials. The NGV is analytically linked to the group refractive index variation of resonant medium.

However, only the material physics approach does not enable to conveniently interpret the NGD effect. Therefore, a typical systemic approach based on the transfer function was introduced [5-8]. By means of the equivalent transfer function, basic topologies of NGD lumped circuit in very low frequencies (VLFs) and in low frequencies (LFs) were proposed in 1990s [7-8]. With such lumped circuits, the NGD signature corresponds to the smoothed signal propagating with output in time advance compared to the corresponding input [7-13]. The asymptotic limits of the NGD effect generated by electronic circuits were reported in [14]. It is noteworthy that the NGD time advance is not in contradiction with the causality [7-10]. Because of this 
counterintuitive aspect, the NGD effect remains one of the most unfamiliar effects ever seen in physics area. Curious design and research engineers in 2000s were wondering on the NGD effect existence in the electronics engineering. Different circuit NGD theoretical approaches and experimentations were carried out to answer to this curiosity [7-14].

In parallel with the metamaterial revolution, very high frequency (VHF) and ultra-high frequency (UHF) NGD microwave circuits were designed in 2000s [15-18]. The NGD effect is induced by material with both negative permittivity and negative permeability or also known as double negative index metamaterials [15-18]. These cases of artificial materials are also known as NGV media [1518]. One of the initial structures allowing to experiment the double negative effect is constituted by split ring resonator (SRR) metamaterial structures [15-18]. Those SRR structures belong to the left-handed periodical circuits. By inspiring with metamaterial-based topologies, transmission line based NGD passive circuits were designed and developed [19-21]. Thanks to the design compactness, tentative applications of NGD circuits were initiated for the improvement of radio frequency (RF) and communication devices as oscillators [22], feedforward amplifiers [23-24], antennas [25-26], non-Foster elements [27-28] and communication device group delay (GD) compensation technique [29-31]. Because of systematic attenuation losses, the performances of NGD passive circuits [15-21] were threatened and less attractive for many electronic engineers. To overcome this critical point of view, some VHF and UHF microwave topologies of NGD active circuits, for example using transistors, were developed [3235].

Despite, the state of the art on the unfamiliar NGD concept, the NGD effect basic interpretation requires further research effort in order to reach higher level of popularity as the classical electronic functions as filter, amplifier, oscillator and attenuators. So far, one of the ways enabling to interpret in more democratic way the NGD concept is the consideration of the analogy between the filter and NGD behaviors [35]. In function of the NGD bandwidth positioning, the innovative concept of bandpass (BP) NGD function was introduced [35]. So far, as the meaning of BP NGD circuit is still not understandable to most of electronic designers and manufacturers. We propose a quite easy to understand and didactical research work about BP NGD circuit engineering opens to nonspecialists as graduated students, technicians and engineers in the present article. The two main bullets of research contributions of the article are:

- A BP NGD characterization of RLC parallel based lumped passive network topology;

- And a special focus on the open question about the robustness of BP characteristic uncertainty analysis (UA) versus the $\mathrm{R}, \mathrm{L}$ and $\mathrm{C}$ component tolerances.
The present research work paper is organized in six different sections as follows:

- Section II defines the main specifications of the unfamiliar BP NGD function. After the introduction of the considered passive topology, the main parameters characterizing the BP NGD responses are analytically defined.

- Section III introduces the BP NGD analysis of the considered passive topology based on RLC parallel (RLCP) passive network. After the frequency response expressions, the NGD parameters will be established in function of the $\mathrm{R}, \mathrm{L}$ and $\mathrm{C}$ ones.

- Section IV develops a figure of merit (FoM) expression of the BP NGD circuit. The BP FoM optimality will be formulated.

- Section $\mathrm{V}$ is focused on the corroborated validation results of calculation, simulation and measurement. The validation of the BP NGD aspect is performed by designing a proof of concept (POC) and PCB prototype composed of lumped components. Then, the BP NGD specifications from analytical calculations, simulations and experimentations are discussed.

- Section VI is innovatively focused on the UA investigation. The aim of this study is to show the influence of quality and tolerance of components for NGD circuit. The UAs of the BP NGD specifications will be literally expressed and numerically verified with respect to the constituting component tolerances.

- Then, Section VII addresses the final conclusion.

\section{FUNDAMENTAL SPECIFICATIONS OF THE UNFAMILIAR BP NGD FUNCTION}

For the better understanding on the unfamiliar BP NGD function, it is necessary to recall the definition of basic BP NGD parameters [35-36]. This preliminary definition is based on the voltage transfer function (VTF) expression of the considered passive topology.

\section{A. DEFINITION OF BP NGD SPECIFICATIONS}

Let us consider the black box of two-port circuit shown in Fig. 1. By denoting the input voltage $V_{i n}$ and the output voltage $V_{\text {out }}$, according to the circuit and system theory, the VTF of the circuit in the frequency domain can be expressed by:

$$
T(j \omega)=\frac{V_{\text {out }}(j \omega)}{V_{\text {in }}(j \omega)}
$$

with the angular frequency complex variable $j \omega$. 


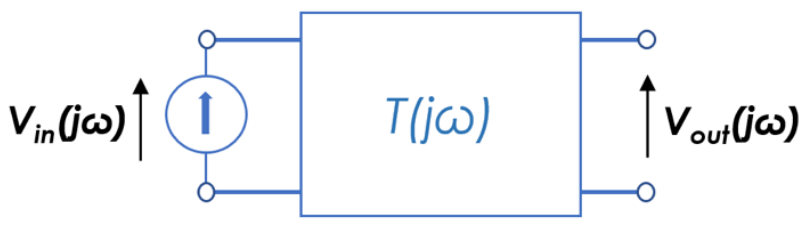

FIGURE 1. Black box diagram with VTF.

The basic elements to explore the BP NGD response of our circuit are the magnitude $T(\omega)$, and the phase, $\psi(\omega)$ which are respectively given by:

$$
\begin{aligned}
& T(\omega)=|T(j \omega)| \\
& \psi(\omega)=\arg [T(j \omega)]
\end{aligned}
$$

According to circuit theory, the GD derived from this last expression is defined by the following equation:

$$
G D(\omega)=-\frac{\partial \psi(\omega)}{\partial \omega}
$$

The BP NGD function depends on the sign of the GD and the frequency band position where it presents negative sign.

\section{B. GRAPHICAL DIAGRAM ILLUSTRATING BP NGD SPECIFICATIONS}

The BP NGD analysis of our RLCP passive topology must be performed with respect to specific parameters linked to the frequency responses. Doing this, we refer the BP NGD specifications with the ideal magnitude and GD diagrams of Figs. 2.

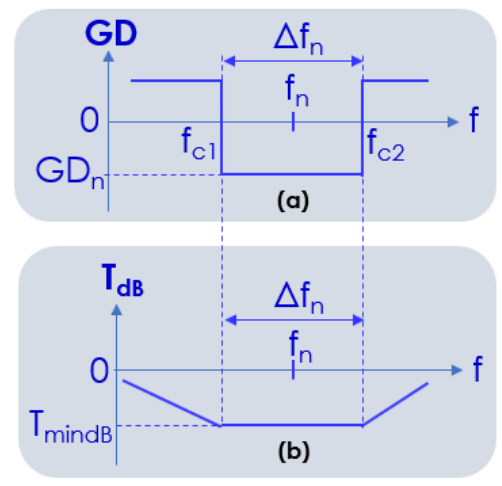

FIGURE 2. Graphical NGD specifications: (a) GD and (b) magnitude.

In this case of study, in difference to most of classical electronics circuit, the main parameters of the characterization are essentially the GD and the magnitude. Subsequently, Fig. 2(a) and Fig. 2(b) introduce ideal plots associated to the GD and magnitude characterizing the unfamiliar BP NGD responses versus positive real frequency traditionally known as:

$$
f=\frac{\omega}{2 \pi}
$$

As illustrated in Fig. 2(a), the ideal GD response of the BP NGD circuit should respect the conditions:

$$
\begin{aligned}
& G D\left(\omega_{c 1}<\omega<\omega_{c 2}\right) \approx G D_{n}<0 \\
& G D\left(\omega<\omega_{1} \cup \omega_{2}<\omega\right)>0
\end{aligned}
$$

In this analytical configuration, we denote the NGD center frequency:

$$
\omega_{n}=2 \pi f_{n}
$$

and the NGD cut-off frequencies:

$$
\omega_{k}=2 \pi f_{k}
$$

with $k=\{1,2\}$. These specific frequencies are related to the GD values following the mathematical equation and inequation defined respectively by:

$$
\begin{aligned}
& G D\left(\omega_{k}\right)=0 \\
& G D\left(\omega_{n}\right)<0
\end{aligned}
$$

The NGD bandwidth is equal to:

$$
\left\{\begin{array}{l}
\Delta \omega_{n}=\omega_{2}-\omega_{1} \\
B W_{N G D}=\frac{\Delta \omega_{n}}{2 \pi}=f_{2}-f_{1}
\end{array}\right.
$$

As seen in Fig. 2(b), in the frequency band, $\omega_{1}<\omega<\omega_{2}$, the attenuation losses must respect the classical constraints related to $T_{\min }$ as classical microwave devices:

$$
|T(j \omega)| \geq T_{\min }
$$

Similar to classical electronic functions as filters, amplifiers, oscillators and many more, the design engineering starting from ideal specification diagrams must be followed by concrete materialization. Therefore, a circuit topology is required.

In this optic, the unfamiliar BP NGD function is concretized by circuit theory of RLCP passive cell introduced in the following section.

\section{BP NGD ANALYSIS OF THE CONSIDERED RLC PARALLEL (RLCP) PASSIVE TOPOLOGY}

The present section develops the VTF based BP NGD analysis of the topology representing lumped passive cell under study. The analytical specification is established in function of the circuit parameters.

\section{A. TOPOLOGICAL INTRODUCTION AND VTF EXPRESSION OF THE RLCP CIRCUIT}

Fig. 2 represents the RLCP network-based topology under study which is represented by passive parallel network comprised of components, resistance, $R_{n}$, inductance, $L_{n}$ and capacitor, $C_{n}$. The proposed passive cell scheme input is connected to voltage source, $V$, corresponding to input voltage, $V_{i n}$. 


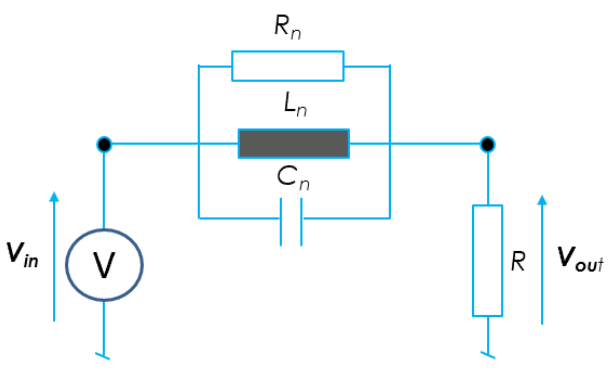

FIGURE 3. RLCP network-based BP NGD passive cell under study.

Moreover, the circuit output, generating output voltage, $V_{\text {out }}$, is connected to shunt resistance, $R$. In brief, we have in the lumped passive cell scheme, input voltage $V_{\text {in }}$ and output voltage $V_{\text {out }}$ across resistance $R$. By means of this scheme, we can define a series complex impedance consisted of $R_{n}$, $L_{n}$ and $C_{n}$ parallel components:

$$
Z(j \omega)=\frac{1}{\frac{1}{R_{n}}+\frac{1}{j \omega L_{n}}+j \omega C_{n}}
$$

Before the analytical development, we can express the VTF is defined by equation (1) by using the voltage divider principle. It can be demonstrated that this VTF can be explicitly rewritten in function of the RLC component parameters by:

$$
T(j \omega)=\frac{R\left[L_{n} \omega+j R_{n}\left(L_{n} C_{n} \omega^{2}-1\right)\right]}{\left(R+R_{n}\right) L_{n} \omega+j R_{n} R\left(L_{n} C_{n} \omega^{2}-1\right)}
$$

From this VTF, we can express the previously introduced NGD characteristics in function of the component parameters in the next subsection.

\section{B. BP NGD CHARACTERISTICS OF THE CONSIDERED PASSIVE TOPOLOGY}

By considering the main quantities of BP NGD frequency responses defined earlier in equations (2), (3) and (4), the magnitude associated to the VTF defined in equation (15) can be given by:

$$
T(\omega)=\frac{R \sqrt{\left(L_{n} \omega\right)^{2}+\left[R_{n}\left(L_{n} C_{n} \omega^{2}-1\right)\right]^{2}}}{\sqrt{\left[\left(R_{n}+R\right) L_{n} \omega\right]^{2}+}}
$$

By definition (3), the corresponding phase response is equal to:

$$
\psi(\omega)=\psi_{1}(\omega)+\psi_{2}(\omega)
$$

with:

$$
\begin{aligned}
& \psi_{1}(\omega)=\arctan \left[\frac{R_{n}}{L_{n} \omega}\left(L_{n} C_{n} \omega^{2}-1\right)\right] \\
& \psi_{2}(\omega)=\arctan \left[\frac{R_{n} R}{L_{n} \omega\left(R_{n}+R\right)}\left(1-L_{n} C_{n} \omega^{2}\right)\right]
\end{aligned}
$$

According to equation (4), the GD is formulated as:

$$
G D(\omega)=G D_{1}(\omega)+G D_{2}(\omega)
$$

with:

$$
\left\{\begin{array}{l}
G D_{1}(\omega)=-\frac{\partial \psi_{1}(\omega)}{\partial \omega} \\
G D_{2}(\omega)=-\frac{\partial \psi_{2}(\omega)}{\partial \omega}
\end{array}\right.
$$

which implies:

$$
\begin{gathered}
G D_{1}(\omega)=\frac{\frac{R_{n}}{L_{n} \omega^{2}}\left(L_{n} C_{n} \omega^{2}-1\right)-2 R_{n} C_{n}}{1+\left[\frac{R_{n}}{L_{n} \omega}\left(L_{n} C_{n} \omega^{2}-1\right)\right]^{2}} \\
G D_{2}(\omega)=\frac{\frac{R_{n} R}{L_{n}\left(R_{n}+R\right) \omega^{2}}\left(1-L_{n} C_{n} \omega^{2}\right)}{+\frac{2 R_{n} R C_{n}}{R_{n}+R}} \\
1+\left[\frac{R_{n} R}{L_{n} \omega\left(R_{n}+R\right)}\left(L_{n} C_{n} \omega^{2}-1\right)\right]^{2}
\end{gathered}
$$

These frequency responses constitute the background of the BP NGD analysis by establishing the basic specifications. The following subsections express these specifications with respect to the $\mathrm{R}, \mathrm{L}$ and $\mathrm{C}$ component parameters.

\section{NGD CENTER FREQUENCY AND BANDWIDTH}

We recall that the NGD bandwidth, denoted, $B W_{N G D}$ is given in relation (12). To determine this parameter, the approach consists of finding the roots of equation (12). The NGD center frequency previously given in equation (21) is also classically known as the resonance frequency:

$$
\omega_{n}=\frac{1}{\sqrt{L_{n} C_{n}}}
$$

of $\mathrm{R}_{n} \mathrm{~L}_{n} \mathrm{C}_{\mathrm{n}}$ network of our passive topology introduced in Fig. 3. This center frequency is linked to the capacitor parameter design equation:

$$
C_{n}=\frac{1}{\sqrt{L_{n} \omega_{n}^{2}}}
$$

Thus, the mathematical simplification leads us to the solutions of the following equation:

$$
L_{n}^{2} C_{n}^{2} \omega^{4}-L_{n}\left(2 C_{n}+\frac{\left(L_{n}\left(R_{n}+R\right)\right)}{R_{n}{ }^{2} R}\right) \omega^{2}+1=0
$$

Therefore, the roots of this equation which represent the cut-off frequencies are:

$$
f_{c_{1}}=\frac{\sqrt{2 C_{n}+\frac{L_{n}\left(R_{n}+R\right)}{R_{n}{ }^{2} R}-}}{2 \pi C_{n} \sqrt{2 L_{n}}}
$$


and:

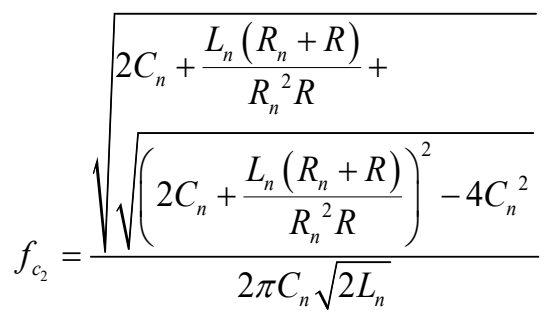

These two frequencies are chosen by supposing:

$$
f_{c_{1}}<f_{c_{2}}
$$

The NGD bandwidth is taken from equation (12) as:

$$
B W_{N G D}=f_{c_{2}}-f_{c_{1}}
$$

At this step, the calculation of the product of the two frequencies makes possible further understanding about symmetrical aspect of the PB NGD GD response. Indeed, from equations (27) and (28), it can be analytically verified that:

$$
f_{c_{1}} f_{c_{2}}=f_{n}^{2}
$$

This last relation proves that our BP NGD response is symmetrical with respect of $f_{n}$.

\section{SPECIFIC NGD PARAMETER EXPRESSIONS AT THE NGD CENTER FREQUENCY}

First of all, we remind that the main goal of the study is to analyze the behavior of the RLCP topology under study around the NGD center frequency. The specifications such as the VTF magnitude and GD can be evaluated in function of this frequency. Therefore, substituting the frequency variable with equation (24) into magnitude given in relation (10), we have:

$$
T_{n}=T\left(\omega_{n}\right)=\frac{R}{R_{n}+R}
$$

Following the same way, we have:

$$
G D_{n}=G D\left(\omega_{n}\right)
$$

By means of the GD expressed in equation (21), the last equation can be detailed as:

$$
G D_{n}=\frac{-2 R_{n}^{2}}{L_{n} \omega_{n}^{2}\left(R_{n}+R\right)}
$$

Then, the NGD bandwidth proposed in equation (30) becomes:

$$
B W_{N G D}=\frac{\sqrt{1+\frac{R_{n}}{R}}}{2 \pi C_{n} R_{n}}
$$

Knowing these BP NGD parameters, we may wonder about the performance criteria. The following section is dedicated to the deep study on the BP NGD figure of merit (FoM).

\section{BP NGD RLCP PASSIVE TOPOLOGY FIGURE OF MERIT}

The present section elaborates the FoM of our BP NGD topology. The mathematical expression of the BP NGD
FoM will be defined. Then, the optimal case will be discussed.

\section{A. BP NGD FIGURE OF MERIT}

To assess the performances of the BP NGD circuit under study, a figure of merit (FoM) has been defined as a product of the circuit NGD parameters previously established at the NGD frequency $f_{n}$. The FoM is expressed by:

$$
F O M_{n}=-T_{n} \cdot B W_{N G D} \cdot G D_{n}
$$

By replacing $B W_{N G D}$ and $G D_{n}$ by their respective expressions at $f_{n}$, the previous formula becomes:

$$
\operatorname{FoM}_{n}=\frac{\left(1-T_{n}\right) \sqrt{T_{n}}}{\pi}
$$

By taking into account the expression of $T_{n}$ at $f_{n}$, we can demonstrate that our FoM depends only on the resistive elements of the RLCP circuit.

\section{B. OPTIMAL VALUE OF BP NGD FOM}

The optimal value of the FoM is obtained from the derivation with respect to the minimal magnitude:

$$
\frac{\partial F_{O} M_{n}}{\partial T_{n}}=0
$$

It can be analytically demonstrated that the derived magnitude optimal value is equal to:

$$
T_{n}=\frac{1}{3}
$$

Substituting this last value into equation (37), we obtain the maximum value of $F_{o M}$ equal to:

$$
\left|F o M_{n}\right|_{\max }=\frac{2}{3 \sqrt{3} \pi}
$$

\section{GRAPHICAL ANALYSIS OF BP NGD FOM}

Fig. 4 represents the respective shapes of $F_{0 M}$ and of the ratio of resistances $R_{n} / R$ as a function of $T_{n}$ at the NGD center frequency $f_{n}$. With respect to the $F_{o} M_{n}$ variational behavior, we identify three areas:

- $\quad T_{n} \in$ ]0; 0.2]: Strong variation of $F_{o M}$ for about one-sixth of the span of $T_{n}$.

- $\left.\left.\quad T_{n} \in\right] 0.2 ; 0.5\right]$ : Weak variation of $F_{o} M_{n}$ for about a third of the span of $T_{n}$.

- $\quad T_{n} \in$ ]0.5; 1[: Significant variation in $F_{0} M_{n}$ over about half the span of $T_{n}$.

Thus, the choice of resistors should be made in zone II of $F o M_{n}$. On the other hand, the resistance ratio can be divided into two areas:

- $\left.\left.\quad T_{n} \in\right] 0 ; 0.03\right]$ : Significant change in $R_{n} / R$ during a very short range of $T_{n}$ (about 1/30).

- $\quad T_{n} \in$ ]0.03; 1 [: Small change in $R_{n} / R$ for about one third of the span of $T_{n}$.

It is found that during the intersection in zone II of $F o M_{n}$ and $R_{n} / R$, the slightest variation in the resistance ratio can induce a remarkable variation in $F_{o M}$. 


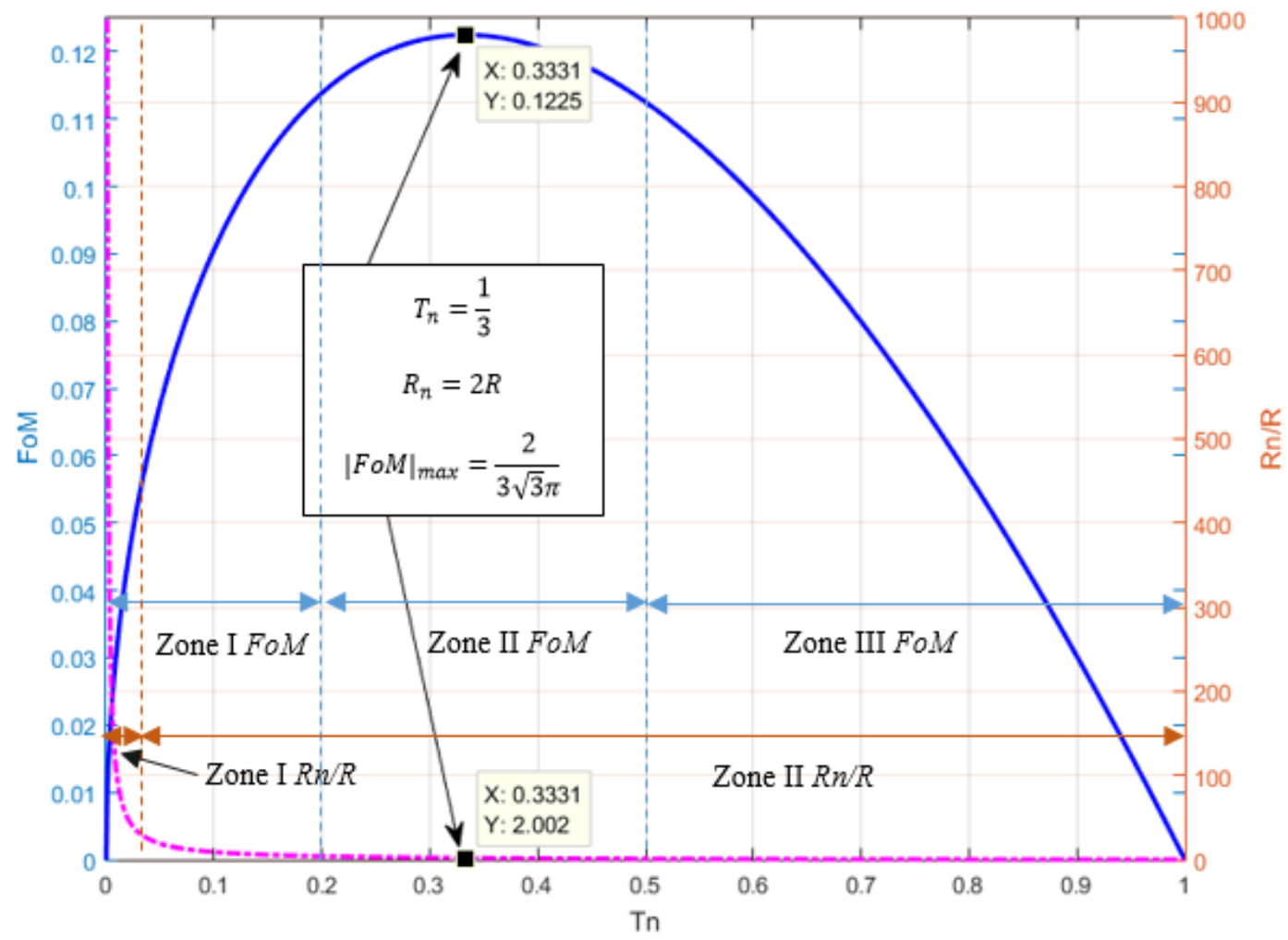

FIGURE 4. FOMn and $R_{n} / R$ ratio variations vs $T_{n}$.

This combination of representation serves to choose relevant values of resistors maximizing FoM $_{n}$.

To verify the validity of the developed BP NGD analysis of RLCP topology, results of POC, prototype demonstrator, simulation and experimentation will be discussed in the following section.

\section{BP NGD BEHAVIOR VALIDATION}

The present section focuses on the BP NGD validation of the RLCP passive topology under study. The frequency

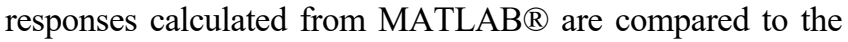
simulation model and the measured prototype.

\section{A. DESIGN DESCRIPTION OF THE BP NGD CIRCUIT POC}

The validation approach is based on the comparison between the MATLAB $\AA$ numerical computation of analytical expressions given in equations (17), (18) and (21) with the simulation and measurement results.

The BP NGD design of the RLCP electronic circuit POC can be performed by the following steps: $\rightarrow$ Step 1: Choice of BP NGD specifications (as indicated in the ideal diagrams of Fig. 2)

$\rightarrow$ Step 2: Calculations of the constituting R, L and C component ideal values constituting the circuit

- Step 3: Choice of available nominal components

$\rightarrow$ Step 4: UA analysis by taking into account the component tolerances.

These different steps of BP NGD circuit can be realized by non-specialist electronic engineering animateurs and also students.

The circuit design was made by choosing the NGD center frequency $f_{n}$ of about $240 \mathrm{MHz}$. As revealed by equation (40), the maximum expression of FoM is reached by choosing resistance values with respect to the relationship $R_{n}$ $=2 R$. After fixing the values of $f_{n}, R_{n}, R$ and $L_{n}$, the value of $C_{n}$ was determined by relation (24).

Table I summarizes the fixed and calculated parameters of the POC RLCP circuit. The frequency domain or AC simulation of VTF was carried out with the commercial tool of electronic circuit simulator $\mathrm{ADS} \otimes$ from Keysight Technologies $\AA$. 
TABLE I. Parameters of the RLCP Circuit POC

\begin{tabular}{cc}
\hline \hline Parameter & Value \\
\hline$f_{n}$ & $240 \mathrm{MHz}$ \\
$R$ & $150 \Omega$ \\
$R_{n}$ & $300 \Omega$ \\
$L_{n}$ & $22 \mathrm{nH}$ \\
$C_{n}$ & $20 \mathrm{pF}$ \\
\hline \hline
\end{tabular}

Fig. 4(a) illustrates the ADS schematic design of the RLCP circuit. Fig. 4(b) displays the fabricated RLCP PCB prototype implemented in hybrid technology. The tested BP NGD PCB is fabricated with $\mathrm{R}, \mathrm{L}$ and $\mathrm{C}$ surface mounted devices (SMDs).
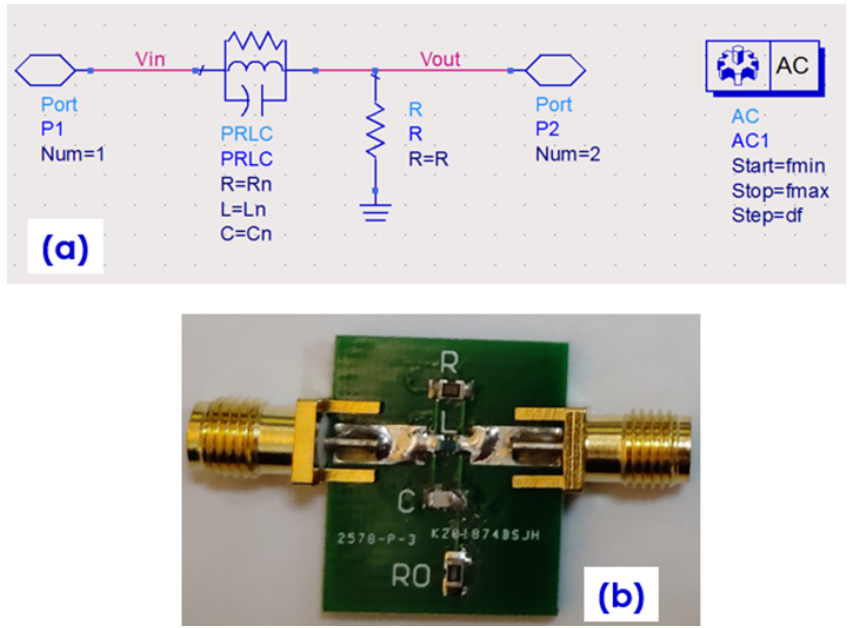

FIGURE 5. (a) POC ADS ${ }^{\circledR}$ design and (b) prototype photograph of NGD passive RLCP circuit.

To validate the BP NGD behavior with the PCB prototype, measurement test campaign was made. The following subsection briefly describes the experimental setup.

\section{B. EXPERIMENTAL INVESTIGATION}

First and foremost, as revealed in the previous design section, our BP NGD demonstrator is implemented as a two-port circuit expected to operate hundred-megahertz frequency range.

We remind that the present experimental investigation is based on the RLCP circuit under test (CUT) shown in Fig. 4(b). The experimentation was performed with S-parameters measurement as proposed in [36]. The photograph of the experimental setup including the BP NGD circuit prototype is illustrated by Fig. 6 . We can see that the test setup of the BP NGD circuit measurement was performed by connecting the circuit input and output to a vector network analyzer (VNA). The employed VNA is referenced Rohde\&Schwarz ${ }^{\circledR}$ ZNB 20 and able to operate in a $100 \mathrm{kHz}$ - $20 \mathrm{GHz}$ frequency band.

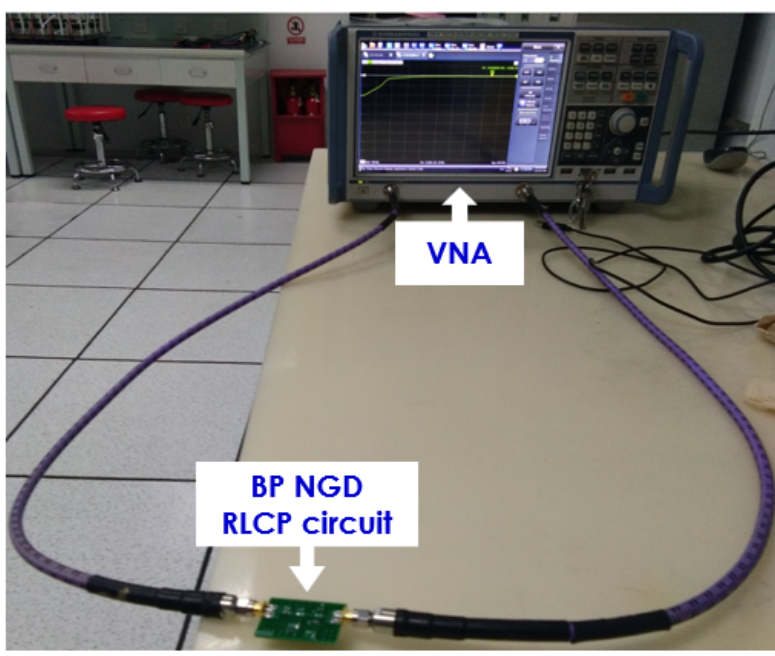

FIGURE 6. Experimental setup photograph of the BP NGD RLCP circuit prototype

As a matter of fact, the obtained validation results of BP NGD responses will be discussed in the following subsection.

\section{COMPARATIVE STUDY OF THE CALCULATED, SIMULATED AND EXPERIMENTED BP NGD RESPONSES}

Table II highlights the expected BP NGD specifications of our demonstrator. In order to verify the BP NGD aspect with the considered POC and demonstrator, the frequency band of the present comparative study is performed within the limits $f_{\min }=140 \mathrm{MHz}$ to $f_{\max }=360 \mathrm{MHz}$.

TABLE II. Targeted BP NGD Specifications

\begin{tabular}{cc}
\hline \hline Parameter & Value \\
\hline$f_{n}$ & $240 \mathrm{MHz}$ \\
$f_{c l}$ & $220.42 \mathrm{MHz}$ \\
$f_{c 2}$ & $261.18 \mathrm{MHz}$ \\
$B W_{N G D}$ & $40.76 \mathrm{MHz}$ \\
$G D_{n}$ & $-10.47 \mathrm{~ns}$ \\
$F o M$ & 0.1224 \\
\hline \hline
\end{tabular}

As results of our BP NGD study, Figs. 7 represent the comparisons of the frequency domain or $\mathrm{AC}$ responses from the POC and demonstrator of RLCP circuit introduced earlier in Figs. 4. The blue solid, cyan dashed and pink dotdashed curves are calculated ("Theory"), simulated ("Simulation") and measured ("Measure") results, respectively. It is plotted in top, middle and bottom of Figs. 7 the magnitude, phase and GD diagrams of our RLCP circuit POC and prototype. We can point out from these plots that the simulated VTF results are very wellcorrelated to the calculated ones. However, notable differences with those two ones are observed for the cases of measurement. Such deviations can be hypothetically explained due: 
- To the PCB prototype fabrication imperfection;

- To the measurement systematic errors as the parasitic effects from connectors, cables and VNA itself;

- And in particular, the uncertainties linked to the R, $\mathrm{L}$, and $\mathrm{C}$ component tolerances in the considered working frequency band.

Table III summarizes the BP NGD specifications as NGD center frequency $f_{n}$, NGD cut-off frequencies $f_{c 1,2}$,
NGD bandwidth $B W$, attenuation $T_{n}$ and NGD value $G D_{n}$ associated to the theoretical, simulated and measured approaches. We can clearly underline by looking at the bottom of Fig. 7 that the considered RLCP topology behaves as a BP NGD function in the working frequency band. The calculated and simulated GD diagrams reveal NGD value of about -10.63 ns while the experimented one is of about $-8.1 \mathrm{~ns}$.
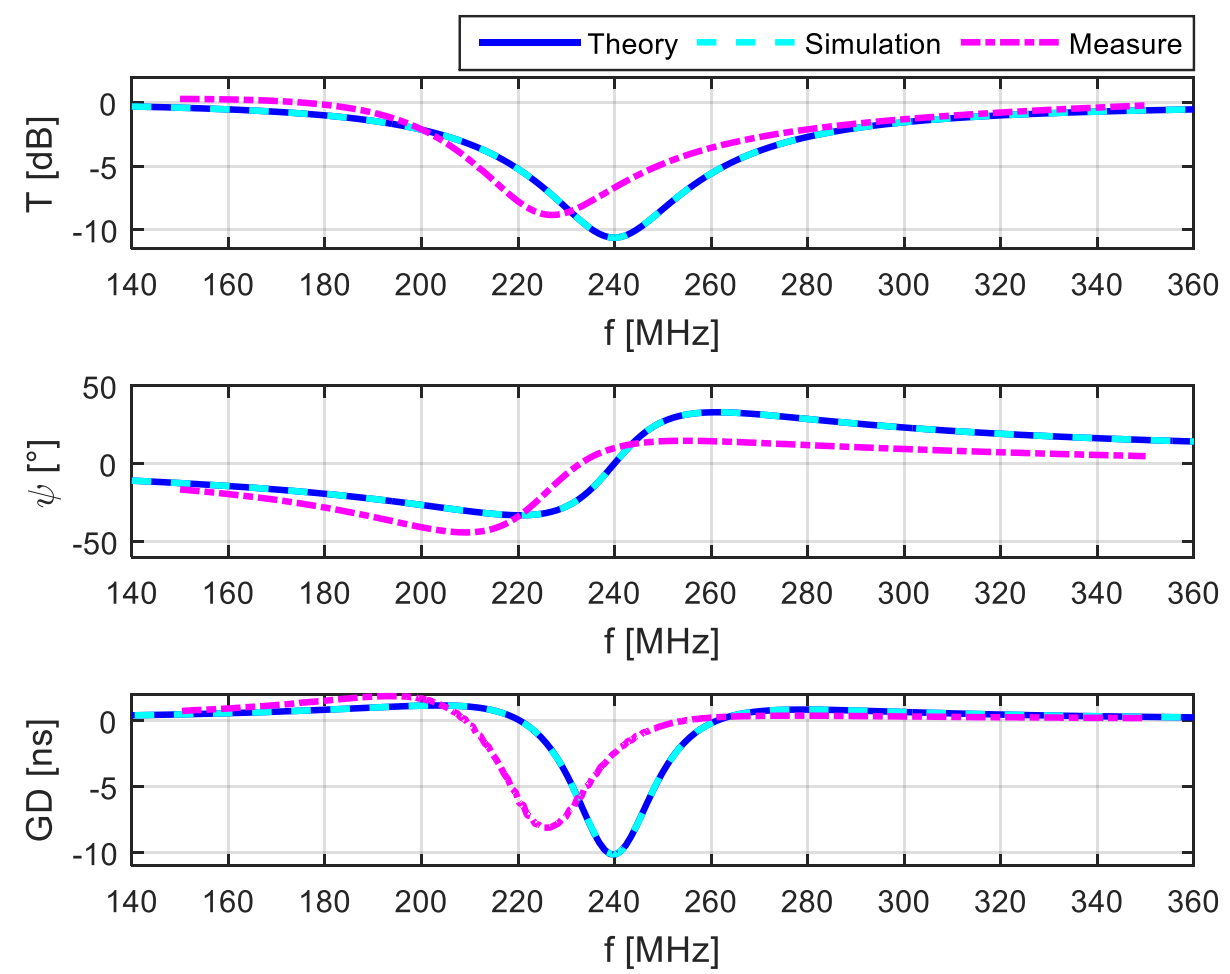

FIGURE 7. Comparison between calculated, simulated and measured (a) magnitudes, (b) phases and (c) GDs of the BP NGD circuit demonstrator shown in Figs. 4.

TABLE III. Comparison between the calculated and simulated NGD parameters

\begin{tabular}{|c|c|c|c|c|c|c|c|}
\hline Approach & $f_{n}(\mathrm{MHz})$ & $f_{c l}(\mathrm{MHz})$ & $f_{c 2}(\mathrm{MHz})$ & $B W(\mathrm{MHz})$ & $T_{n}(\mathrm{~dB})$ & $G D_{n}(\mathrm{~ns})$ & FoM \\
\hline Theory & 240.1 & 219.8 & 261.6 & 41.8 & -10.629 & -10.155 & 0.1248 \\
\hline Simulation & 239.6 & 218.7 & 259.4 & 41.3 & -10.63 & -10.15 & 0.1232 \\
\hline Measurement & 227.1 & 209 & 253.2 & 44.2 & -8.84 & -8.115 & 0.1055 \\
\hline $\begin{array}{c}\text { Relative error (\%) } \\
\text { between theory vs } \\
\text { simulation }\end{array}$ & 0.21 & 0.50 & 0.84 & 1.20 & 0.01 & 0.05 & 1.28 \\
\hline $\begin{array}{c}\text { Relative error (\%) } \\
\text { between theory vs } \\
\text { measurement }\end{array}$ & 5.41 & 4.91 & 3.21 & 5.74 & 16.83 & 20.09 & 15.46 \\
\hline
\end{tabular}


Moreover, we can point out that the simulated $F_{0 M}$ is much closer to the calculated one with $1.28 \%$ maximum shift. Furthermore, the measured results have discrepancies from $5 \%$ to $15.5 \%$.

Based on such finding of deviations, open questions are raised about the sensitivity of the BP NGD specifications in function of the component tolerances. This curious finding is answered in the present study by means of UA. The BP NGD performance sensitivity linked to the imperfections of the $\mathrm{R}, \mathrm{L}$ and $\mathrm{C}$ component fabrication is materialized in the next section via theoretical approaches and comparison with $\mathrm{ADS} \AA$ parametric $\mathrm{AC}$ simulations.

\section{UA OF THE BP NGD CIRCUIT WITH RESPECT TO} THE R, L AND C COMPONENT IMPERFECTIONS

In the present section, the UA regarding the $\mathrm{R}, \mathrm{L}$ and $\mathrm{C}$ component imperfections by means of theoretical and simulation approach will be investigated. Doing this, the analytical expressions of the NGD parameters with respect to lumped $\mathrm{R}, \mathrm{L}$, and $\mathrm{C}$ tolerances are established. Then, the numerical validations are discussed.

\section{A. THEORETICAL EXPRESSIONS OF THE BP NGD SPECIFICATIONS UA}

Due to the fabrication imperfection, it is well known that passive components can undergo variations depending on their nature using conditions, the mathematical variations:

$$
\Delta x=\left\{\Delta R, \Delta R_{n}, \Delta L_{n}, \Delta C_{n}\right\}
$$

are considered respectively on each passive component constituting the NGD cell. Computations were carried out using the principle of maximizing margins of error. It is noteworthy that our approach considers the absolute value of the inputs that each component involved in the NGD specifications, $f_{n}, T_{n}, G D_{n}$ and $B W_{n}$. By considering the previously established quantities expressed in equations (5), (17), (18) and (21), the analytical expressions of the NGD specification UAs are given as follows:

$$
\begin{gathered}
\frac{\Delta f_{n}}{f_{n}} \leq \frac{\frac{\Delta L_{n}}{L_{n}}+\frac{\Delta C_{n}}{C_{n}}}{2} \\
\frac{\Delta T_{n}}{T_{n}} \leq \frac{R_{n}}{R_{n}+R}\left(\frac{\Delta R}{R}+\frac{\Delta R_{n}}{R_{n}}\right) \\
\frac{\Delta G D_{n}}{G D_{n}} \leq\left(\frac{R}{R_{n}+R}\right) \frac{\Delta R}{R}+\left(\frac{R_{n}+2 R}{R_{n}+R}\right) \frac{\Delta R_{n}}{R_{n}}+\frac{\Delta L_{n}}{L_{n}} \\
\frac{\Delta B W_{n}}{B W_{n}} \leq \frac{1}{2}\left(\frac{R_{n}}{R_{n}+R}\right) \frac{\Delta R}{R}+\left(\frac{R_{n}+2 R}{2\left(R_{n}+R\right)}\right) \frac{\Delta R_{n}}{R_{n}}+\frac{\Delta C_{n}}{C_{n}} \\
\frac{\Delta F o M_{n}}{F o M_{n}} \leq \frac{1}{2} \mid \frac{R_{n}+2 R}{R_{n}+R}\left(\frac{\Delta R}{R}+\frac{\Delta R_{n}}{R_{n}}\right)
\end{gathered}
$$

Relations (42) to (46) are commonly published in the literature, especially considering uncertainty management methods such as GUM (Guide to the expression of uncertainty in measurement) [37]. To verify the relevance of these analytical formulas, numeric applicative studies with relative tolerances denoted $\Delta x / x$ equal to $1 \%, 2 \%$ and $5 \%$ are proposed in the next paragraph.

\section{B. BP NGD PARAMETERS UA ON A VARIATION RANGE OF RLC COMPONENTS VALUES}

This subsection analyses the 2-D mapping behavior of the BP NGD parameters. For each component, we consider the variation range:

- From $1 \mathrm{~m} \Omega$ to $1 \mathrm{k} \Omega$ for $R_{n}$,

- From $1 \mathrm{~m} \Omega$ to $1 \mathrm{k} \Omega$ for $R$,

- And from $1 \mathrm{nH}$ to $50 \mathrm{nH}$ for $L_{n}$ (which is also equivalent to $C_{n}$ ).

Figs. 8, 9, and 10 represent the $\mathrm{ADS} ®$ simulated results of VTF magnitude, phase, and GD mappings with respect to both frequency and each $R_{n}, R, L_{n}$ and $C_{n}$ elements.

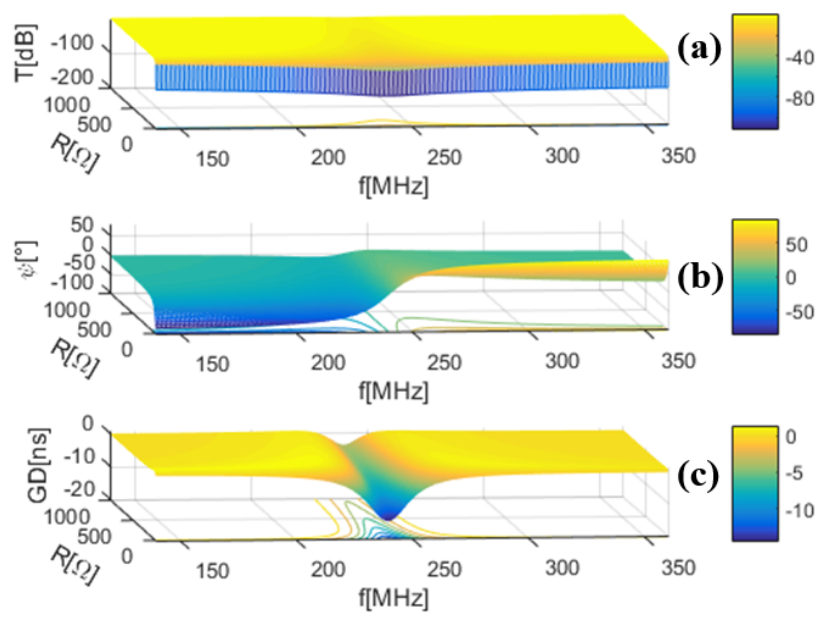

FIGURE 8. Variations of the VTF magnitude, phase and GD with respect to resistance $R$ variation in the range from $1 \mathrm{~m} \Omega$ to $1 \mathrm{k} \Omega$.
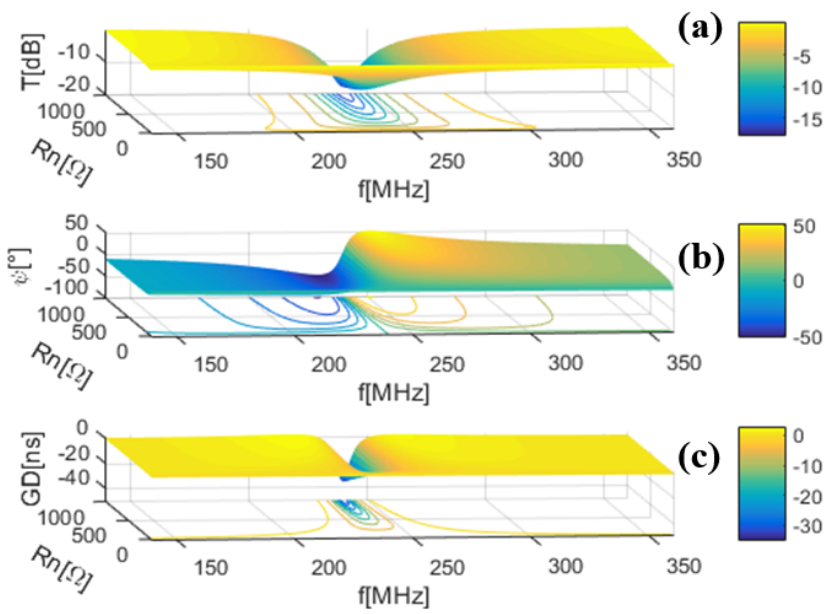

FIGURE 9. Variations of the VTF magnitude, phase and GD with respect to resistance $R_{n}$ variation in the range from $1 \mathrm{~m} \Omega$ to $1 \mathrm{k} \Omega$. 

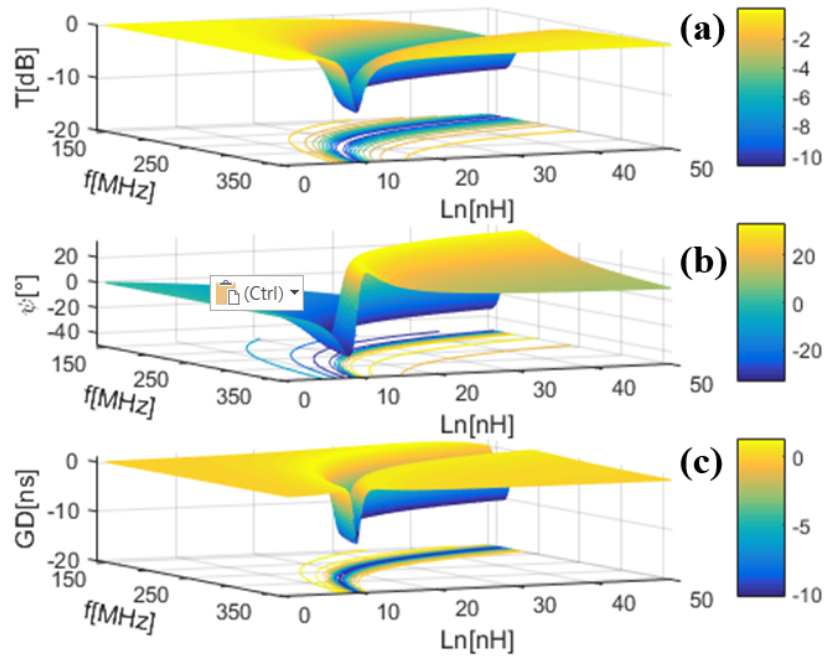

FIGURE 10. Variations of the VTF magnitude, phase and GD with respect to inductance $L_{n}$ variation in the range from $1 \mathrm{nH}$ to $50 \mathrm{nH}$.

From these 2-D plots, we can point up that:

- In Fig. 8(a) and 8(b), we can remark the less influence of the output resistance $R$ on $T(\omega)$ and $\psi$ (in ${ }^{\circ}$ ). While, as illustrated in Fig. 8(c), the GD tends to increase with the output resistance $R$.

- Fig. 9 represents the cartographies of the NGD parameters versus frequency and $R_{n}$. The magnitude (resp. GD) tends to decrease when $R_{n}$ values are in its lower range as seen in Fig. 9(a) (resp. 9(c)).

- We emphasize from Fig. 10, we can denote that the NGD parameters decrease when $L_{n}$ also decreases.

\section{NUMERIC APPLICATION}

The present UA numeric application consolidates the BP NGD performance variation and sensitivity in function of $R, L$ and $C$ component fabrication imperfections. The present UA applicative study is based on the comparison between the calculated and simulated results.

\section{1) UA CALCULATED RESULTS}

Table IV, Table $\mathrm{V}$ and Table VI highlight the influence of tolerances of $R, R_{n}$, and $L$ (or $C$ ) element values on each BP NGD parameters. We consider in the present case of study the relative tolerances $1 \%, 2 \%$ and $5 \%$.

TABLE IV. UAs of NGD specifications according to variations of $R$

Uncertainty [\%]

\begin{tabular}{ccccccc} 
& \multicolumn{2}{c}{$R \pm 1 \%$} & \multicolumn{2}{c}{$R \pm 5 \%$} \\
\hline Specification & Theory & ADS ${ }^{\circ}$ & Theory & ADS & Theory & ADS \\
$T_{n}$ & 0.706 & 0.608 & 1.412 & 1.361 & 3.529 & 3.401 \\
$G D_{n}$ & 0.294 & 0.296 & 0.588 & 0.591 & 1.471 & 1.478 \\
$B W_{n}$ & 0.353 & 0 & 0.706 & 0 & 1.765 & 2.392 \\
$F o M$ & 0.647 & 0.383 & 1.294 & 0.761 & 3.235 & 0.564 \\
\hline \hline
\end{tabular}

TABLE V. UAs of NGD specifications according to variations of $R_{n}$

\section{Uncertainty [\%]}

\begin{tabular}{ccccccc} 
& \multicolumn{2}{c}{$R_{n} \pm 1 \%$} & \multicolumn{3}{c}{$R_{n} \pm 2 \%$} \\
\hline Specification & Theory & ADS $®$ & Theory & ADS® & Theory & ADS® \\
$T_{n}$ & 0.706 & 0.680 & 1.412 & 1.361 & 3.529 & 3.401 \\
$G D_{n}$ & 1.294 & 1.281 & 2.588 & 2.562 & 6.471 & 6.502 \\
$B W_{n}$ & 0.647 & 2.392 & 1.294 & 2.392 & 3.235 & 5.263 \\
$F o M$ & 0.647 & 1.815 & 1.294 & 1.254 & 3.235 & 2.535 \\
\hline \hline
\end{tabular}




\begin{tabular}{|c|c|c|c|c|c|c|}
\hline \multirow[b]{3}{*}{ Specification } & \multicolumn{6}{|c|}{ Uncertainty [\%] } \\
\hline & \multicolumn{2}{|c|}{$L_{n}$ or $C_{n} \pm 1 \%$} & \multicolumn{2}{|c|}{$L_{n}$ or $C_{n} \pm 2 \%$} & \multicolumn{2}{|c|}{$L_{n}$ or $C_{n} \pm 5 \%$} \\
\hline & Theory & $\operatorname{ADS} \bowtie$ & Theory & $\mathrm{ADS} \circledast$ & Theory & $\mathrm{ADS} \otimes$ \\
\hline$f_{n}$ & 0.5 & 0.458 & 1 & 0.916 & 2.5 & 2.291 \\
\hline$T_{n}$ & 0 & 0 & 0 & 0 & 0 & 0 \\
\hline$G D_{n}$ & 1 & 0.099 & 2 & 0.099 & 5 & 0.099 \\
\hline$B W_{n}$ & 1 & 0.239 & 2 & 2.632 & 5 & 5.263 \\
\hline FoM & 0 & 0.141 & 0 & 2.536 & 0 & 5.170 \\
\hline
\end{tabular}

The following remarks can be emphasized from the numerical application:

- $\quad$ Table IV and Table V show that the $T_{n}$ uncertainty depends only to the resistive parameters $\left(R\right.$ and $\left.R_{n}\right)$ but not to the reactive components. We can underline that $R$ and $R_{n}$ have the same influence on the uncertainty of $T_{n}$. A $5 \%$ relative tolerance on $R$ and $R_{n}$, induces a maximum uncertainty of VTF magnitude less than $3.53 \%$.

- It is important to recall that in difference to the case of $f_{n}$, as pointed out in equation (25), the NGD center frequency $f_{n}$ depends only on the $L$ and $C$ parameters. It means that the $f_{n}$ UA is only in function of the $L$ and $C$ tolerances. As seen in Table VI, we remark that when the relative tolerances are fixed to $5 \%$, the $f_{n} \mathrm{UA}$ is around $2.5 \%$.

- The NGD value $G D_{n}$ appears to be the most sensitive NGD parameters to the variations of the lumped elements. A 5\% relative tolerance on $R$ and $R_{n}$ implies respectively relative variation more than $1.4 \%$, and $6.5 \%$ around the GD. While a $2 \%$ relative tolerance on $L$ and $C$ elements generates an uncertainty of $2 \%$ around the GD.

- The observation of Table VI, allows to notice that the NGD bandwidth and FoM are also sensitive to the variation of each circuit component. A 5\% relative tolerance of the inductance and the capacitance causes an uncertainty more than $5 \%$ around the NGD bandwidth and FoM.

\section{2) DISCUSSION ON THE UA CALCULATED AND SIMULATED RESULTS}

The previous numeric applications on UA revealed the effect of RLCP POC and prototype lumped component tolerances. The variations of the BP NGD parameters enable to quantify the sensitivity in function of the component fluctuation.

We noticed from the different calculations:

- How the NGD parameter uncertainties vary with respect to the tolerances. Furthermore, we can also predict in certain cases that the NGD parameter relative variations are not changing non-linearly. In some cases, they appear to change following hyperbolic variation.

- It is also noticed that $G D_{n}, B W_{n}$ and $F o M$ parameters are more sensible to the $R, R_{n}, L_{n}$ and $C_{n}$ component imperfections. Under relative tolerance of $5 \%$, the variation of $R_{n}$ induces more impact on all NGD parameters than the other lumped elements. However, it is important to precise that the proposed UA of RLCP circuit BP NGD parameters is done by considering $\mathrm{R}, \mathrm{L}$ and $\mathrm{C}$ components changing independently.

- Of course, at this stage of study, most design electronic engineers may ask about the undesirable effects on the BP NGD parameters related to the superposition of tolerances of all lumped components. This curious question constitutes our ongoing future research work, we plan to study simultaneous variation of several parameters by means of equations (42) to (46).

\section{CONCLUSION}

An UA of the BP NGD passive topology ever being done before is developed. The considered topology is essentially comprised of RLCP-network cell.

The BP NGD circuit theory is established from VTF in function of frequency variation and RLC passive circuit elements. The analytical BP NGD characterization of the NGD specifications in function of the $\mathrm{R}, \mathrm{L}$ and $\mathrm{C}$ component values is developed. The main specifications of the NGD circuit as the NGD center frequency, NGD value, NGD bandwidth, attenuation and even the BP NGD FoM were investigated. The analytical expressions of the NGD specifications have been established. It was demonstrated how the BP NGD FoM is maximized for a certain value of the resistances of the RLCP NGD circuit. This optimal FoM corresponds to $1 / 3$ attenuation (approximately $-9.54 \mathrm{~dB}$ ).

Three different approaches based on theoretical, simulation and experimental validations were undertaken. 
The simulation was carried out with the ADS ${ }^{\circledR}$ commercial tool. The relevance of the BP NGD theory is validated with excellent agreement with simulations and measurements.

The UA investigations show that the BP NGD circuit attenuation is sensitive to resistance variations. The same behavior is found for the NGD value and NGD bandwidth but they are literally sensitive to reactive components variations. The performed UAs enable to understand how sensitive is our BP NGD topology with respect to the component tolerances.

In the future, the present BP NGD investigation could contribute in particular to the improvement of the electronic and communication system by:

- Compensating the transmission signal delay [22,2931],

- Reducing undesirable resonance effect against PCB electromagnetic compatibility (EMC) issues [38-39],

- And to design frequency independent NGD phase shifter [40].

To develop such a BP NGD circuit application, we can take care on the UA with the used component imperfection considerations.

\section{ACKNOWLEDGMENT}

The authors would like to thank Dr. Blaise Ravelo from NUIST, China for his advice and scientific support on the bandpass (BP) NGD circuit study.

\section{REFERENCES}

[1] S. Chu and S. Wong, "Linear Pulse Propagation in an Absorbing Medium," Phys. Rev. Lett., Vol. 48, 1982, pp. 738-741.

[2] B. Ségard and B. Macke, "Observation of Negative Velocity Pulse Propagation," Phys. Lett. A, Vol. 109, 1985, pp. 213-216.

[3] B. Macke and B. Ségard, "Propagation of light-pulses at a negative group-velocity," Eur. Phys. J. D, Vol. 23, 2003, pp. 125-141.

[4] J. N. Munday and W. M. Robertson, "Observation of Negative Group Delays within a Coaxial Photonic Crystal Using an Impulse Response Method," Optics Communications, Vol. 273, No. 1, 2007, pp. 32-36.

[5] B. Ségard and B. Macke, "Two-pulse interference and superluminality," Optics Communications, Vol. 281, No. Jan. 2008, pp. $12-17$.

[6] B. Macke, B. Ségard, and F. Wielonsky, "Optimal superluminal systems," Phys. Rev. E, Vol. 72, No. 3, 035601(R), 2005, pp. 1-3.

[7] M. W. Mitchell, and R. Y. Chiao, "Negative group delay and "fronts" in a causal system: An experiment with very low frequency bandpass amplifiers," Phys. Lett. A, vol. 230, no. 3-4, June 1997, pp. 133-138.

[8] M. W. Mitchell and R.Y. Chiao, "Causality and Negative Groupdelays in a Simple Bandpass Amplifier," Am. J. Phys., vol. 66, 1998, pp. 14-19.

[9] T. Nakanishi, K. Sugiyama and M. Kitano, "Demonstration of Negative Group-delays in a Simple Electronic Circuit," Am. J. Phys., vol. 70 , no. 11,2002 , pp. 1117-1121.

[10] M. Kitano, T. Nakanishi and K. Sugiyama, "Negative Group-delay and Superluminal Propagation: An Electronic Circuit Approach," IEEE J. Sel. Top. in Quantum Electron., vol. 9, no. 1, Feb. 2003, pp. 43-51.

[11] K.-P. Ahn, R. Ishikawa, A. Saitou and K. Honjo, "Synthesis for Negative Group Delay Circuits Using Distributed and Second-Order RC Circuit Configurations," IEICE Trans. on Electronics, Vol. E92C, No. 9, 2009, pp. 1176-1181.
[12] J. N. Munday and R. H. Henderson, "Superluminal Time Advance of a Complex Audio Signal," Appl. Phys. Lett., vol. 85, no. 3, July 2004, pp. 503-504.

[13] F. Wan, J. Wang, B. Ravelo, J. Ge, and B. Li, "Time-Domain Experimentation of NGD Active RC-Network Cell," IEEE Trans. Circuits and Systems II: Express Briefs, Vol. 66, No. 4, Apr. 2019, pp. 562-566.

[14] M. Kandic and G. E. Bridges, "Asymptotic Limits of Negative Group Delay in Active Resonator-Based Distributed Circuits, ” IEEE Transactions on Circuits and Systems I: Regular Papers, Vol. 58, No. 8, Aug. 2011, pp. 1727-1735.

[15] G. V. Eleftheriades, O. Siddiqui, and A. K. Iyer, "Transmission Line for Negative Refractive Index Media and Associated Implementations without Excess Resonators," IEEE Microw. Wireless Compon. Lett., Vol. 13, No. 2, pp. 51-53, Feb. 2003.

[16] O. F. Siddiqui, M. Mojahedi and G. V. Eleftheriades, "Periodically Loaded Transmission Line With Effective Negative Refractive Index and Negative Group Velocity," IEEE Trans. Antennas Propagat., Vol. 51, No. 10, Oct. 2003, pp. 2619-2625.

[17] L. Markley and G. V. Eleftheriades, "Quad-Band NegativeRefractive-Index Transmission-Line Unit Cell with Reduced Group Delay," Electronics Letters, Vol. 46, No. 17, Aug. 2010, pp. 12061208.

[18] G. Monti and L. Tarricone, "Negative Group Velocity in a Split Ring Resonator-Coupled Microstrip Line," Progress In Electromagnetics Research, Vol. 94, pp. 33-47, 2009.

[19] G. Liu, and J. Xu, "Compact transmission-type negative group delay circuit with low attenuation," Electronics Letters, Vol. 53, No. 7, Mar. 2017, pp. 476-478.

[20] G. Chaudhary, and Y. Jeong, "Tunable center frequency negative group delay filter using coupling matrix approach," IEEE Microwave Wireless Component Letters, Vol. 27, No. 1, 2017, pp. 37-39.

[21] T. Shao, S. Fang, Z. Wang and H. Liu, "A Compact Dual-Band Negative Group Delay Microwave Circuit," Radio Engineering, vol. 27, no. 4, pp. 1070-1076, Dec. 2018.

[22] C. D. Broomfield and J. K. A. Everard, "Broadband Negative Group Delay Networks for Compensation of Oscillators, Filters and Communication Systems," Electron. Lett., Vol. 36, No. 23, pp. 19311933, Nov. 2000.

[23] H. Choi, Y. Jeong, C. D. Kim, and J. S. Kenney, "Efficiency enhancement of feedforward amplifiers by employing a negative group delay circuit," IEEE Trans. Microw. Theory Tech., Vol. 58, No. 5, May 2010, pp. 1116-1125.

[24] H. Choi, Y. Jeong, C. D. Kim, and J. S. Kenney, "Bandwidth enhancement of an analog feedback amplifier by employing a negative group delay circuit," Progress In Electromagnetics Research, Vol. 105, 2010, pp. 253-272.

[25] A. Mortazawi and W. Alomar, "Negative group delay circuit," United States Patent Application US20160093958, Mar. 2016.

[26] M. Zhu and C.-T. M. Wu, "Reconfigurable Series Feed Network for Squint-free Antenna Beamforming Using Distributed AmplifierBased Negative Group Delay Circuit," in Proc. 2019 49th European Microwave Conference (EuMC), Paris, France, 1-3 Oct. 2019, pp. 256-259.

[27] H. Mirzaei and G. V. Eleftheriades, "Realizing non-Foster reactive elements using negative-group-delay networks," IEEE Trans. Microw. Theory Techn., vol. 61, no. 12, pp. 4322-4332, Dec. 2013.

[28] T. Zhang, R. Xu and C. M. Wu, "Unconditionally Stable Non-Foster Element Using Active Transversal-Filter-Based Negative Group Delay Circuit," IEEE Microw. Wireless Compon. Lett., vol. 27, no. 10, pp. 921-923, Oct. 2017.

[29] T. Shao, Z. Wang, S. Fang, H. Liu and Z. N. Chen, "A group-delaycompensation admittance inverter for full-passband self-equalization of linear-phase band-pass filter," Int. J. Electron. Commun., Vol. 123, No. 153297, 2020, pp. 1-6.

[30] K.-P. Ahn, R. Ishikawa and K. Honjo, "Group Delay Equalized UWB InGaP/GaAs HBT MMIC Amplifier using Negative Group Delay Circuits," IEEE Trans. Microw. Theory Techn., Vol. 57, No. 9 , Sept. 2009, pp. 2139- 2147.

[31] B. Ravelo, S. Lalléchère, A. Thakur, A. Saini and P. Thakur, "Theory and circuit modelling of baseband and modulated signal 
delay compensations with low- and band-pass NGD effects", Int. J. Electron. Commun., Vol. 70, No. 9, Sept. 2016, pp. 1122-1127.

[32] B. Ravelo, "Investigation on microwave negative group delay circuit", Electromagnetics, Vol. 31, No. 8, Nov. 2011, pp. 537-549.

[33] C.-T. M. Wu and T. Itoh, "Maximally Flat Negative Group Delay Circuit: A Microwave Transversal Filter Approach," IEEE Trans. on Microwave Theory and Techniques, Vol. 62, No. 6, June 2014, pp. 1330-1342.

[34] F. Wan, N. Li, B. Ravelo, Q. Ji, B. Li and J. Ge, "The Design Method of the Active Negative Group Delay Circuits Based on a Microwave Amplifier and an RL-series Network," IEEE ACCESS, Vol. 6, Dec. 2018, pp. 33849-33858.

[35] B. Ravelo, "Similitude between the NGD function and filter gain behaviours," Int. J. Circ. Theor. Appl., Vol. 42, No. 10, Oct. 2014, pp. 1016-1032.

[36] B. Ravelo, S. Ngoho, G. Fontgalland, L. Rajaoarisoa, W. Rahajandraibe, R. Vauché, Z. Xu, F. Wan, J. Ge, and S. Lalléchère, "Original Theory of NGD Low Pass-High Pass Composite Function for Designing Inductorless BP NGD Lumped Circuit,” IEEE Access, Vol. 8, No. 1, Oct. 2020, pp. 192951-192964.

[37] GUM: BIPM, IEC, IFCC, ILAC, ISO, IUPAC, IUPAP and OIML, 2008 Guide to the Expression of Uncertainty in Measurement, JCGM 100:2008, GUM 1995 with minor corrections.

[38] B. Ravelo, F. Wan, J. Nebhen, W. Rahajandraibe, and S. Lalléchère, "Resonance Effect Reduction with Bandpass Negative Group Delay Fully Passive Function", IEEE Transactions on Circuits and Systems II: Express Briefs, Vol. 68, No. 7, July 2021, pp. 2364-2368.

[39] B. Ravelo, S. Lalléchère, W. Rahajandraibe, and F. Wan, "Electromagnetic Cavity Resonance Equalization with Bandpass Negative Group Delay," IEEE Transactions on Electromagnetic Compatibility, Vol. 63, No. 4, Aug. 2021, pp. 1248-1257.

[40] B. Ravelo, G. Fontgalland, H. S. Silva, J. Nebhen, W. Rahajandraibe, M. Guerin, G. Chan, and F. Wan, "Original Application of StopBand Negative Group Delay Microwave Passive Circuit for TwoStep Stair Phase Shifter Designing," IEEE Access, Vol. 10, No. 1, 2022, pp. 1493-1508. 